\title{
PERFIL DOS RECÉM-NASCIDOS VIVOS EM HOSPITAL UNIVERSITÁRIO DE ALTA COMPLEXIDADE DO MUNICÍPIO DE PORTO ALEGRE/RS
}

\author{
PROFILE OF ALIVE NEWBORNS IN A UNIVERSITY HOSPITAL OF \\ HIGH COMPLEXITY OF THE CITY OF PORTO ALEGRE/RS
}

\section{Carla Skilhan de Almeida}

Curso de Fisioterapia

Universidade Federal do Rio Grande do Sul

\section{Alessandra Bombarda Müller}

Curso de Fisioterapia

Universidade do Vale dos Sinos

Laís Rodrigues Gerzson

Programa de Pós-Graduação em Saúde da

Criança e do Adolescente

Universidade Federal do Rio Grande do Sul

\author{
Nádia Cristina Valentini \\ Programa de Pós-Graduação em Ciências do \\ Movimento Humano \\ Universidade Federal do Rio Grande do Sul
}

Contato

Carla Skilhan de Almeida

E-mail: carlaskilhan@gmail.com

\section{RESUMO}

Objetivo: No intuito de identificar a população de neonatos assistidos em um hospital universitário de alta complexidade de Porto Alegre/RS, buscou-se descrever o perfil dos recém-nascidos (RN) vivos, considerados típicos, no primeiro semestre de 2012. Métodos: estudo retrospectivo, descritivo, transversal, cuja amostra foi aleatória, incluindo 152 neonatos atendidos na maternidade do hospital uma vez por semana. As informações foram transferidas dos prontuários e/ou avaliações fisioterapêuticas e digitadas em um banco de dados. Foram transcritos os dados relativos ao sexo da criança, perímetro cefálico, comprimento e peso ao nascer, tempo de gestação, APGAR no $5^{\circ}$ minuto e idade da mãe com a finalidade de realizar uma análise descritiva dos achados para possíveis planejamentos de ações de promoção e prática da saúde comunitária. Resultados: Neste estudo, 51,32\% eram meninas, embora no Brasil nasçam mais crianças do sexo masculino. A idade gestacional foi com uma média de 38,7 semanas. O peso dos RN teve média de $3.382 \mathrm{~g}$. Quanto ao APGAR no $5^{\circ}$ minuto, todos apresentam acima de 7 . A idade materna média foi de 27,8 anos. Quanto aos aspectos ao nascer, as crianças apresentam uma média de 49,5 cm de comprimento e $34,5 \mathrm{~cm}$ de perímetro cefálico. Com relação ao tipo de parto, encontrou-se uma prevalência de $70,39 \%$ de cesáreas. Conclusão: Políticas de saúde dirigidas para a redução de cesáreas e para melhor acesso aos serviços atenção pré-natal, em prol de oferecer a promoção e a prevenção da saúde para nossas crianças, são necessárias.

Palavras-chave: Recém-nascido; Perfil de saúde; Hospital universitário. 


\section{ABSTRACT}

Aim: In order to identify the population of newborns assisted at a university hospital of high complexity of Porto Alegre / RS, this research attempted to describe the profile of alive newborns, considered normal, in the first half of 2012. Methods: A retrospective, descriptive cross-study, whose random sample including 152 newborns treated at the maternity hospital once a week. The information was transferred from medical records and / or physical therapy reviews and typed into a database. The data transcribed included the sex of the child, head circumference, length and birth weight, gestational age, Apgar score at the $5^{\text {th }}$ minute, and mother's age, in order to conduct a descriptive analysis of the findings for possible planning of promotion and practice community health. Results: In this study, $51.32 \%$ were girls, although it is more common that male children are born in Brazil. Gestational age was an average of 38.7 weeks. The weight of newborns averaged $3.382 \mathrm{~g}$. As for the $5^{\text {th }}$ minute APGAR were all above 7 . The average maternal age was 27.8 years. As for the aspects during birth, the newborn children have an average of $49.5 \mathrm{~cm}$ of length and $34.5 \mathrm{~cm}$ head circumference. Regarding the type of labor, there was a prevalence of $70.39 \%$ of c-sections. Conclusions: It is necessary to promote health policies directed to c-section reduction and for better access to prenatal care services, in order to provide the promotion and prevention of health care for our children.

Keywords: Infant, newborn; Health profile; Hospitals, university.

\section{INTRODUÇÃO}

As ações de saúde pública interdisciplinares são fundamentais para a promoção da saúde, por meio da redução das taxas de mortalidade e morbidade materna e infantil e da diminuição das diferenças socioeconômicas ${ }^{1}$. As condições de saúde dos Recém-Nascidos (RN) vivos podem ser analisadas mediante vários parâmetros, dos quais um dos mais estudados, hoje em dia, é o peso ao nascimento ${ }^{2}$. Essa medida é função da massa corpórea, cuja constituição é o resultado de um processo complexo para o qual concorrem inúmeros fatores de origem biológica, social e ecológica. Encontramos vários estudos sobre fatores potencialmente determinantes do peso ao nascer, como o sexo do RN, sua etnia, a estatura e a idade dos pais, a situação socioeconômica familiar, a escolaridade materna, os nascimentos múltiplos, a duração da gestação, a paridade, o intervalo entre os partos, a história obstétrica anterior, cuidados pré-natais, o ganho de peso e a morbidade materna durante a gravide $z^{3}$.

Dados epidemiológicos caracterizando a população de RN no Brasil podem ser obtidos desde 1990, quando foi implantado, no Ministério da Saúde, o Sistema de Informações sobre Nascidos Vivos (SINASC), por meio de um documento individualizado e padronizado, em nível nacional - a Declaração de Nascido Vivo -, que contempla a obtenção desses dados fundamentais, indispensáveis para 0 planejamento e decisão política na área de saúde materno-infantil ${ }^{4}$.

É importante conhecer o que existe efetivamente na saúde das comunidades, tanto do ponto de vista socioeconômico quanto do 
sanitário para que se possa planejar, atuar e, posteriormente, reavaliar a situação do RN, permitindo detectar se as mudanças almejadas foram alcançadas. Nesse processo, utilizamse indicadores demográficos, epidemiológicos, político-sociais e econômicos, entre outros, a fim de permitir uma descrição mais próxima da situação real.

Relativo aos eventos vitais, o conhecimento do número de RN vivos de determinado local em um período é imprescindível, pois ele é componente de vários índices e coeficientes, além de fundamentar o planejamento de ações na área materno-infantil ${ }^{5}$. Em caso de $\mathrm{RN}$ vivos sem complicações, é importante conhecer seu perfil epidemiológico para, se necessário, acompanhar seu desenvolvimento global. Quando o RN apresenta injúrias ao nascimento, é necessária sua permanência em Unidade de Terapia Intensiva Neonatal (UTI-NEO). Caso focalize-se especificamente ao desenvolvimento motor deste neonato, sabe-se que a intervenção motora é quase que inerente ao tratamento. Já em neonatos considerados típicos, defrontamos, muitas vezes, com a dificuldade na detecção de possíveis desvios motores, sendo observados tardiamente, ou quando os atrasos motores já estão estabelecidos.

Para melhor classificar os $\mathrm{RN}$ em relação à variável peso ao nascimento, adota-se a seguinte definição: RN de muito baixo peso $(<1.500 \mathrm{~g}), \mathrm{RN}$ de baixo peso $(<2.500 \mathrm{~g})$ e RN com peso acima de $2.500 \mathrm{~g}^{6}$. Com relação à idade gestacional, a classificação descrita atualmente é: nascimento pré-termo (até a $37^{\mathrm{a}}$ semana de gestação), nascimento à termo (entre a $38^{\mathrm{a}}$ e a $42^{a}$ semanas de gestação) e nascimento póstermo (após a $42^{\mathrm{a}}$ semana de gestação) ${ }^{7}$. O que a literatura relata como padrão são neonatos com peso ao nascimento entre $2.500 \mathrm{~g} \mathrm{e}<4.000$ $\mathrm{g}^{8}$, com perímetro cefálico entre $34 \mathrm{~cm}$ e $35 \mathrm{~cm}^{9}$, idade gestacional maior que 37 semanas ${ }^{10}$, estatura entre $49 \mathrm{~cm}$ e $50 \mathrm{~cm}^{9}$ índice de APGAR de 7 a 10 no quinto minuto ${ }^{11}$.

Os estudos publicados na área da fisioterapia trazem perfis de $\mathrm{RN}$ com algum tipo de risco, ou seja, que inserem dados de neonatos de risco com baixo peso ao nascimento, índice de APGAR temerário, nascimentos precoces e provenientes de famílias com condições socioeconômicas desfavoráveis. A importância do conhecimento do perfil dos neonatos típicos para o profissional que atua diretamente com essa população preconiza uma visão preventiva da assistência ${ }^{12}$, bem como para alunos de graduação que têm um primeiro contato com estes RN.

Dessa forma, no intuito de identificar a população de neonatos RN nos seus primeiros dias de vida, assistidos por um grupo de estudantes de fisioterapia em um hospital universitário de alta complexidade de Porto Alegre/RS, buscou-se descrever o perfil dos RN vivos, considerados típicos, no primeiro semestre de 2012.

\section{MÉTODOS}

Estudo descritivo e observacional, retrospectivo, com delineamento transversal, cuja população foi composta por RN vivos do Hospital São Lucas da Pontifícia Universidade Católica do Rio Grande do Sul (HSL-PUCRS), no período de janeiro a junho de 2006. Foi aprovado pelo Comitê de Ética em Pesquisa da PUCRS ( $n^{\circ}$ 05/02788) conforme normas estabelecidas pela Resolução 466/2012 do Conselho Nacional de Saúde e ocorreu somente 
após a assinatura do Termo de Consentimento Livre e Esclarecido (TCLE) pelos participantes. A amostra foi aleatória, incluindo 152 neonatos considerados típicos, atendidos na maternidade do hospital, avaliados pelos alunos da Faculdade de Fisioterapia da PUCRS, uma vez por semana. A avaliação consistia em registrar os dados de peso e altura ao nascimento, sexo, índice de APGAR no $5^{\circ}$ minuto, tipo de parto realizado, idade gestacional e idade da mãe. Cabe sinalizar que a avaliação consistia também nos registros do comportamento do neonato em respostas aos estímulos, não sendo aqui objeto de estudo. A inserção dos participantes excetuava neonatos encaminhados para a UTI-NEO. Foram excluídos os neonatos que apresentaram complicações nesse período de internação.

Os neonatos nascidos no centro obstétrico eram posteriormente encaminhados com a mãe para o alojamento conjunto. Os alunos de graduação do curso de fisioterapia iam ao alojamento e realizavam uma sequência de questões à mãe, consequentemente, avaliavam o RN. Todos os dados eram descritos em uma Ficha de Avaliação com antecedentes obstétricos da mãe, idade e tipo de parto, data de nascimento do RN, peso, sexo, idade gestacional do RN, índice de APGAR. Após, os RN eram avaliados neurologicamente quanto aos seus reflexos primitivos, tônus, sinais de anormalidades (tremor, clônus) e conduta (irritabilidade e consolo) movimentos gerais, sensibilidade visual e auditiva. Esses dados neurológicos não estão contemplados neste artigo. As informações coletadas para este estudo baseavam-se na Declaração de Nascidos-Vivos fornecida pelo hospital. Os dados eram tabulados pelo professor responsável em um banco de dados, organizados e analisados no programa estatístico Software Statistical Package for the Social Sciences (SPSS) versão 21.0. Foram utilizadas estatisticamente as medidas de tendência central com os dados obtidos, bem como caracterização da amostra estratificada.

\section{RESULTADOS}

No primeiro semestre de 2006, nasceram 1.204 crianças no HSL-PUCRS; e neste estudo, 152 crianças foram avaliadas pelos alunos da Faculdade de Fisioterapia da PUCRS. Os dados coletados estão apresentados na tabela 1. 
Tabela 1: Distribuição das variáveis e frequência de ocorrência.

\begin{tabular}{|c|c|c|}
\hline Variáveis & $\mathbf{N}$ & $\%$ \\
\hline \multicolumn{3}{|l|}{ Sexo } \\
\hline Masculino & 74 & 48,68 \\
\hline Feminino & 78 & 51,32 \\
\hline Tdade Gestacional (sem) & 38,7 (média) & \\
\hline$<37$ & 13 & 8,55 \\
\hline $37-41$ & 130 & 85,53 \\
\hline$>41$ & 09 & 5,92 \\
\hline Peso ao nascer (g) & 3.382 (média) & \\
\hline \multicolumn{3}{|l|}{ APGAR $5^{\circ}$ minuto } \\
\hline $0-3$ & - & - \\
\hline $4-6$ & - & - \\
\hline $7-10$ & 152 & 100,00 \\
\hline Tdade materna (anos) & 27,8 (média) & \\
\hline Comprimento $(\mathrm{cm})$ & 49,5 (média) & \\
\hline Perímetro cefálico $(\mathrm{cm})$ & 34,5 (média) & \\
\hline \multicolumn{3}{|l|}{ Tipo de parto } \\
\hline Cesáreo & 45 & 29,61 \\
\hline Normal & 107 & 70,39 \\
\hline
\end{tabular}

\section{DISCUSSÃO}

A análise das características de $\mathrm{RN}$ vivos de determinada região é um importante instrumento para o conhecimento do perfil epidemiológico dessa população porque pode fornecer subsídios para o planejamento da promoção da saúde, sempre que necessário, bem como medidas interventivas focalizadas nas variáveis deficitárias, como é o caso do baixo peso ao nascimento, frequentemente encontrado nas crianças brasileiras, principalmente aquelas inseridas em condições socioeconômicas desfavoráveis ao desenvolvimento familiar ${ }^{13}$.

Quanto ao sexo dos RN, no Brasil, nascem mais crianças do sexo masculino, cerca de $2,5 \%$ a mais que crianças do sexo feminino. Em 2010, dos 2.861 .868 nascidos vivos, $51,3 \%$ foram do sexo masculino; e 48,7\%, do sexo feminino ${ }^{14}$. Em estudo realizado em São Paulo ${ }^{15}$, foram encontrados $52,2 \%$ de meninos; neste estudo, $48,68 \%$ meninos e $51,32 \%$ meninas.

Investigando as relações com a idade gestacional, inúmeros estudos relatam a ocorrência de prematuridade associada ao baixo peso do $\mathrm{RN}$ e à pouca instrução materna. Os fatores que têm demonstrado maior influência para um futuro atraso no desenvolvimento são condições socioeconômicas baixas, fraco nível intelectual dos pais e prematuridade ${ }^{16}$. Na família, pode ser a separação parental, desentendimento crônico, alcoolismo, doença crônica e óbito ${ }^{17}$. 
A gestação da mulher tem duração média de 280 dias, considerada a partir do primeiro dia da última menstruação até o dia do parto. Isso resulta em um período gestacional de 40 semanas aproximadamente ${ }^{18}$. Acredita-se que cerca de $5 \%$ a $15 \%$ dos bebês no Brasil nascem abaixo de 37 semanas de idade gestacional ${ }^{19}$. Observamos, na amostra estudada, uma média de 38,7 semanas de idade gestacional, ou seja, 273 dias.

Estudos realizados também enfatizaram as repercussões da prematuridade no crescimento e desenvolvimento de crianças. Concluiu-se que os aspectos sociais e a prematuridade são fatores de risco que influenciam negativamente no desenvolvimento neuropsicomotor ${ }^{16,20}$. Além disso, foram encontradas fortes evidências de que crianças nascidas com baixo peso mostraram mais chances de terem dificuldades físicas e cognitivas no seu desenvolvimento posterior ${ }^{21}$.

De acordo com Lubchenco ${ }^{22}$, a importância do peso ao nascimento, assim como da idade gestacional, na previsão de problemas em RN, justifica-se pelo papel relevante que estes desempenham na maturidade de vários sistemas, tanto nas crianças nascidas pré-termo quanto a termo. Portanto, peso ao nascimento e idade gestacional são variáveis relacionadas; e possíveis desvios de qualquer um dos parâmetros para fora da faixa de normalidade resultarão em aumento da morbidade.
Como neste estudo observou-se o neonato que não necessitou de cuidados intensivos, apenas $8,55 \%$ dos RN do HSLPUCRS estavam abaixo de 37 semanas de gestação (13 bebês). Na comparação com estudos prévios, este índice é muito maior (até $15,3 \%$ ), pois muitos consideram os bebês que necessitam de cuidados intensivos ${ }^{23}$.

No estudo de Santos ${ }^{15}, 93,6 \%$ dos partos encontravam-se entre 37 semanas e 41 semanas de gestação. Neste estudo, 85,53\% dos neonatos apresentaram idade gestacional normal. No Brasil, nascimentos ocorridos na $37^{a}$ e na $38^{a}$ semanas foram de $10 \%$ e $22 \%$ dos nascimentos, em 2012, respectivamente. As variações regionais desses percentuais são mais marcantes na $38^{a}$ semana $(19 \%$ no Norte e no Nordeste e $24 \%$ nas demais regiões) ${ }^{24}$. Esses resultados estão adequados aos números oficiais brasileiros, que indicam as populações das regiões Centro-Oeste, Sudeste e Sul como aquelas que, historicamente, têm maiores índices de desenvolvimento, mais acesso aos serviços básicos de educação, saneamento e saúde e, nos últimos anos, maior redução da taxa de natalidade ${ }^{25}$.

Em um estudo na região Nordeste, foram entrevistadas 3.009 puérperas, sendo que 10,9\% apresentaram idade gestacional $<37$ semanas. As análises apontaram que o risco social (baixa adesão ao pré-natal, localização geográfica, entre outros), associado ao risco biológico 
(idade, primigestação e prematuridade), possa ter interferido na prevalência de baixo peso entre conceptos de mães jovens nessa região. Inclusive, chama a atenção o elevado percentual de cesarianas e da prematuridade que tem impacto na saúde materno-infantili ${ }^{26}$.

$O$ índice de APGAR é considerado um indicador diretamente relacionado com a qualidade da assistência no momento do parto. Apesar da influência das condições prévias do $\mathrm{RN}$, ainda no ambiente intrauterino, que determinam a vitalidade no momento do nascimento, uma má assistência, em que ocorra sofrimento fetal, pode implicar um índice baixo, mesmo em gestações a termo com peso adequado $^{27}$.

Pesquisas médicas ${ }^{12}$ incluem como fatores de risco para índice de APGAR baixo a idade materna avançada ou precoce, fatores de ordem social (estado civil, grau de escolaridade e dúvida sobre a data da última menstruação), história obstétrica (gestações prévias, ocorrência de abortos), assistência pré-natal, complicações clínicas e obstétricas (pré-eclâmpsia, ameaça de parto prematuro, idade gestacional, peso e sexo do RN). Neste estudo, todos os 152 neonatos observados receberam índices de APGAR acima de 7, provavelmente devido à inclusão somente dos bebês que não necessitaram de assistência e cuidados intensivos na UTI-NEO.

A redução da mortalidade infantil, embora com níveis elevados de morbidade, tem possibilitado intervenções cada vez mais na esfera dos serviços de saúde, especialmente, dos médico-assistenciais ${ }^{1}$. Os avanços na assistência prestada a $\mathrm{RN}$ de alto risco do ponto de vista biológico têm contribuído para o aumento significativo dos índices de sobrevivência dessas crianças. Assim, o grande diferencial socioeconômico entre as gestantes reflete-se, hoje, na assistência de boa qualidade ao parto, e o índice de APGAR assume importância crescente como indicador de risco.

A prática da cesariana, ao contrário de ser protetora do índice de APGAR baixo, pode ser fator de risco, quando o número de cesáreas ultrapassa o número predito de cesáreas. $O$ Ministério da Saúde editou, em 1998, a Portaria 2.816, que limita a proporção de cesáreas a serem pagas pelo Sistema Único de Saúde (SUS) (valores recomendados pela Organização Mundial de Saúde entre $10 \%$ e 15\%, com base em indicações clínicas) ${ }^{28}$. A cesárea é um recurso que deve ser utilizado quando o desenrolar normal do parto implica algum tipo de risco para a mãe, para o feto ou para ambos. Como todo procedimento cirúrgico, a cesárea não é isenta de riscos, estando associada a uma maior morbimortalidade materna e infantil ${ }^{29}$.

A média brasileira é de $52,34 \%$

cesáreas $^{30}, \mathrm{e}$, considerando a amostra estudada inserida em um hospital universitário que atende pelo SUS, encontramos ainda assim um elevado índice de partos cirúrgicos: 45 cesáreas 
$(29,61 \%)$ e 107 partos normais $(70,39 \%)$. Fazse necessária uma investigação das razões de tal inversão de valores e a implementação de ações que estimulem a prática mais frequente de partos espontâneos.

Muitos estudos têm demonstrado relações elevadas entre taxas de cesáreas e padrão socioeconômico ${ }^{31}$. Esses achados apontam para a incoerência de encontrar uma maior frequência de partos operatórios justamente na população com melhor padrão aquisitivo, que teoricamente apresentaria um menor risco gestacional e necessitaria menos deste tipo de intervenção. Entretanto, os valores entre a população mais desfavorecida não podem ser considerados ideais, uma vez que são apenas mais baixos. Esses trabalhos indicam que a cesárea parece ter-se tornado um bem de consumo, dependente do poder aquisitivo da população e uma distorção da prática médica.

Este estudo evidenciou prevalências relativamente baixas de neonatos prematuros, com peso médio ao nascimento e índice de APGAR adequados, achados diretamente relacionados com a inclusão na amostra somente de neonatos considerados típicos, o que difere de grande parte das pesquisas acompanhadas, que inserem dados de neonatos de risco, ou seja, aqueles com baixo peso ao nascimento, índice de APGAR temerário, precoces e provenientes de famílias com condições socioeconômicas desfavoráveis. Esta análise revelou também elevada prevalência de partos cesáreos, fator passível de intervenção em um prazo relativamente curto que pode trazer resultados positivos para a saúde materna e infantil.
Além do acima exposto, o estudante de fisioterapia pode ter um primeiro contato com bebês típicos no início de seu curso de formação. Isso traz ao estudante o conhecimento do típico como construção de seu alicerce para o atípico e para futuras discussões.

Há a necessidade de políticas de saúde, especialmente dirigidas para a redução de cesáreas e para a melhoria da cobertura e acesso aos serviços atenção pré-natal. Acreditamos que as autoridades públicas podem oferecer melhores oportunidades para um bom começo de vida para as nossas crianças, avançando na descentralização do sistema de saúde e protegendo a mulher grávida com estratégias intersetoriais de inclusão social. É preciso não esquecer, entretanto, que é à esfera local que compete o interesse, a análise e a utilização dessas informações, no sentido de identificar os principais problemas de saúde na área maternoinfantil apontados a partir do conhecimento do perfil de neonatos e de desenvolver as ações específicas necessárias.

Informações sobre mortalidade e morbidade, o conhecimento sobre os determinantes socioeconômicos e culturais do processo saúde-doença, a atenção à distribuição desigual das doenças no espaço urbano e rural, o contato com os usuários dos serviços de saúde e a comunidade local podem permitir o planejamento necessário de forma a sanar grande parte dos problemas de saúde da população. 


\section{CONCLUSÃO}

Este estudo revelou aos $\mathrm{RN}$ estudados um índice dentro dos padrões de normalidade para: equiparação de sexo, bebês a termo, peso ao nascimento dentro dos padrões, índice de APGAR, comprimento e perímetro cefálico. Esta análise revelou também elevada prevalência de partos cesáreos. Estudantes de fisioterapia puderam ter um primeiro contato com bebês típicos no início de sua formação.

\section{REFERÊNCIAS}

1. Basso CG, Neves ET, Silveira A. Associação entre realização de pré-natal e morbidade neonatal. Texto Contexto Enferm 2012; 21(2):269-76.

2. Costa RS, Caldevilla DE, Gallo PR, Sena BF, Leone C. Incidence and Characteristics of Insufficient Birth Weight Newborns from a Cohort of Neonates in a Public Regional Hospital of a Metropolitan Area. J. Hum. Growth Dev 2013; 23(2):238-243.

3. Fernandes MP, Bierhals IO, Demoliner F, Pretto ADB, Pastore CA. Fatores maternos associados ao peso ao nascer em gestantes de baixo risco obstétrico de uma maternidade-escola do sul do Brasil. Nutr clín diet. Hosp 2014; 34(3):48-56.

4. Guimarães PV, Coeli CM, Cardoso RCA, Medronho RDA, Fonseca SC, Pinheiro RS. Reliability of data from a very low birth weight population in the Live Birth Information System 2005-2006. Rev bras epidemiol 2012; 15(4):694-704.

5. Pedraza DF. Qualidade do Sistema de Informações sobre Nascidos Vivos (Sinasc): análise crítica da literatura. Cien Saude Colet 2012;17(10):2729-2737.

6. Neto PGF, Falcão MC. Cronologia de erupção dos primeiros dentes decíduos em crianças nascidas prematuras com peso inferior a 1500g. Rev Paul Pediatr 2014; 32(1):17-23.

7. Pinheiro RC, Martinez CMS, Fontaine AMGV. Integração viso motora e desenvolvimento global de crianças pré-termo e a termo no início da escolarização. Rev. bras. crescimento desenvolv. Hum 2014; 24(2):181-187.

8. Sousa MACA, Guimarães ICB, Daltro C, Guimarães AC. Association between birth weight and cardiovascular risk factors in adolescents. Arq Bras Cardiol 2013; 101(1):09-17.

9. WHO Multicentre Growth Reference Study Group. WHO Child Growth Standards: Growth velocity based on weight, length and head circumference: Methods and development. Geneva: World Health Organization; 2009.

10. Ersdal HL, Linde J, Mduma E, Auestad B, Perlman J. Neonatal outcome following cord clamping after onset of spontaneous respiration. Pediatrics 2014; 134(2):265-272.

11. Oliveira TG, Freire PV, Moreira FT, Moraes JS, Arrelaro RC, Rossi S, et al. Apgar score and neonatal mortality in a hospital located in the southern area of São Paulo city, Brazil. Einstein 2012; 10(1):22-28.

12. Stokes M. Neurologia para fisioterapeutas. São Paulo: Premier; 2000. 
13. Araújo BBM, Rodrigues BMRD, Araújo PST. A promoção do cuidado materno ao neonato prematuro: a perspectiva da educação problematizadora em saúde [Promoting mothers' care for premature neonates: the perspective of problem-based education in health]. Rev Latino-Am Enfermagem 2015; 23(1):128-131.

14. Brasil. Ministério da Saúde. Secretaria de Atenção à Saúde. Departamento de Ações Programáticas Estratégicas. Atenção à saúde do recém-nascido: guia para os profissionais de saúde / Ministério da Saúde, Secretaria de Atenção à Saúde, Departamento de Ações Programáticas Estratégicas. - 2. ed. - Brasília: Ministério da Saúde; 2012.

15. Santos JDO, Pacheco TS, Oliveira PSD, Pinto VL, Gabrielloni MC, Barbieri M. The obstetrical and newborn profile of postpartum women in maternities in São Paulo. R. pesq.: cuid. fundam. online. 2015; 7(1):1936-1945.

16. Pilatti I, Haas T, Sachetti A, Fontana C, Oliveira SG, Schiavinato JCC. Oportunidades para o desenvolvimento motor infantil em ambientes domésticos. Rev Bras Ciên Saúde 2011;9(27):2227.

17. Pagliarin KC, Brancalioni AR, Keske-Soares M, Souza APR. Relationship among severity of phonological disorders and familial factors. Revista CEFAC 2011; 13(3):414-427.

18. Camargo LA, Pellicciari CR, Novo JLVG, Novo NF. Antropometria e aspectos neonatais de recém-nascidos de gestações de 41 a 42 semanas. Rev Fac Ciênc Méd Sorocaba 2014; 16(4):182-187.

19. Ferreira APA, Albuquerque RC, Rabelo ARM, Farias FC, Correia RCB, Gagliardo HGRG, et al. Comportamento visual e desenvolvimento motor de recém-nascidos prematuros no primeiro mês de vida. Rev bras crescimento desenvolv hum 2011; 21(2):335-343.

20. Pinheiro RC, Martinez CMS, Fontaine AMGV. Visual motor integration and overall development of preterm and at term children at the beginning of schooling. Rev bras crescimento desenvolv hum 2014; 24(2):181-187.

21. Oliveira GE, Magalhães LC, Salmela LFT. Relationship between very low birth weight, environmental factors, and motor and cognitive development of children of 5 and 6 years old. Rev bras fisioter $2011 ; 15(2): 138-145$. 
22. Lubchenco LO. Determinação do peso e idade gestacional. Rev. Médica e Científica, 1984. p. 207-227,

23. Als $\mathrm{H}$, Gilkerson $\mathrm{L}$. The role of relationship-based developmentally supportive newborn intensive care in strengthening outcomes of preterm infants. Seminary of Perinatology. 1997; 21: 178-189.

24. Brasil. Ministério da Saúde. Secretaria de Vigilância em Saúde. Departamento de Análise de Situação em Saúde. Saúde Brasil 2013: uma análise da situação de saúde e das doenças transmissíveis relacionadas à pobreza / Ministério da Saúde, Secretaria de Vigilância em Saúde, Departamento de Análise de Situação em Saúde. - Brasília: Ministério da Saúde; 2014.

25. Monteiro NA. Desigualdades regionais no Brasil: notas sobre o padrão de intervenção do Estado nos anos 2000-2010. In: Guimarães, Paulo Ferraz; Aguiar, Rodrigo Almeida De; Lastres, Helena Maria Martins; Silva, Marcelo Machado da (Org.). Um olhar territorial para o desenvolvimento: Nordeste. Rio de Janeiro: Banco Nacional de Desenvolvimento Econômico e Social; 2014.

26. Almeida AHDVD, Costa MCO, Gama SGND, Amaral MTR, Vieira GO. Low weight at birth in adolescent and young adult mothers in the Northeast Region of Brazil. Rev bras saúde matern Infant 2014; 14(3):279-286.

27. Lima EDFA, Sousa AI, Griep RH, Primo CC. Risk factors for neonatal mortality in the city of Serra, Espírito Santo. Rev Bras Enferm 2012; 65(4):578-585.

28. Laurenti R, Jorge MHPDM, Gotlieb SLD, Oliveira BZD, Pimentel EC. The study of the motherchild binomium: description and general results. Rev bras epidemiol 2015; 18(2):398-412.

29. Velho MB, Santos EKA, Collaço VS. Natural childbirth and cesarean section: social representations of women who experienced them. Rev Bras Enferm 2014; 67(2):282-289.

30. Ministério da Saúde. Departamento de Informática do SUS. Indicadores e dados básicos do Brasil. Brasília: Ministério da Saúde; 2010.

31. Robles AF. From the" risk pregnancy" to the" risk maternity". Biopolitic and health regulation in working class women experience in Recife, Brazil. Physis 2015; 25(1):139-169. 